\title{
Four ways to determine the electron density in low-temperature plasmas
}

\author{
R. F. G. Meulenbroeks, M. F. M. Steenbakkers, Z. Qing, M. C. M. van de Sanden, and D. C. Schram \\ Department of Physics, Eindhoven University of Technology, P.O. Box 5135600 MB, Eindhoven, The Netherlands
}

(Received 8 November 1993)

Four ways to measure the electron density in low-temperature plasmas are presented: Thomson scattering, Langmuir probe, optical-emission spectroscopy, and continuum-radiation analysis. The results of the four methods are compared to each other and discussed. For the electron-density range of $10^{19}-10^{21} \mathrm{~m}^{-3}$, Thomson scattering proved to give the most accurate results (within a few percent); the Langmuir-probe measurements also proved acceptable (25\%). A collisional-radiative-model fit through excited-level populations and continuum analysis yields results in good agreement with Thomson scattering data, although with larger margins of error (around 40\%). A simple Saha fit proved to be inadequate.

PACS number(s): 52.70.Kz, 52.40.Hf, 52.25.Rv

\section{INTRODUCTION}

In a sense, the study of plasmas begins with measuring $n_{e}$, the electron density. It constitutes one of the most fundamental parameters for plasmas, as for many plasma types $n_{e}$ varies a great deal more than, e.g., the electron temperature $T_{e}$. However, it is often difficult to determine accurately $n_{e}$ (say, within $10 \%$ ). In the present study, we present four methods to determine $n_{e}$ in lowtemperature (electron temperature below $0.5 \mathrm{eV}$ ) plasmas.

Probably the most accurate, local, and unambiguous way to determine $n_{e}$ (in our experiment: $n_{e}>10^{18} \mathrm{~m}^{-3}$ ) is Thomson scattering [1]. Accuracies of $3 \%$ can be achieved using this diagnostic; the tradeoffs are price and complexity [2]. In the present study we compare other (cheaper and more simple) methods, i.e., Langmuir double probe, optical-emission spectroscopy (OES), and continuum analysis, to Thomson scattering. The measurements are carried out on a low-pressure recombining plasma jet, which has been used successfully for deposition of carbon and silicon materials $[3,4]$ and looks promising for source applications $\left(\mathrm{H}^{+}, \mathrm{H}^{-}\right)$[5] as well (Fig. 1). With its greatly varying $n_{e}[2,6]$ it also proved very suitable as a subject for this investigation.

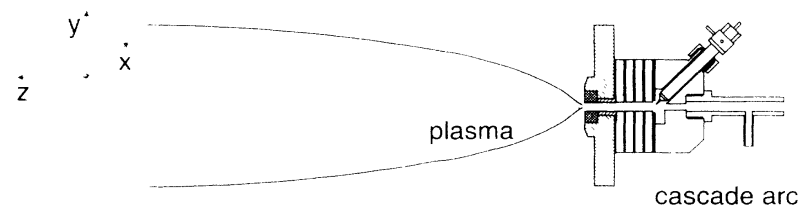

FIG. 1. The cascaded arc experiment. A thermal plasma at subatmospheric pressure is created in a continuously operated $\mathrm{dc}$ arc, consisting of three cathodes, three electrically isolated copper plates, and an anode plate. All the parts are water cooled. The plasma expands through the nozzle into a lowpressure $(40 \mathrm{~Pa})$ vessel, creating a supersonically expanding plasma jet.

\section{EXPERIMENT AND DIAGNOSTICS}

A continuously operated dc arc creates an argon plasma at subatmospheric pressures $(0.6-0.2 \mathrm{bar}, 4-\mathrm{mm}$-diam plasma channel) which expands into a heavily pumped vacuum vessel, creating a supersonic expansion, ending in a stationary shock and followed by a subsonic relaxation. The plasma source can be moved within the vacuum vessel (in the $x, y$, and $z$ directions). The vacuum vessel has a length of $3 \mathrm{~m}$ and a diameter of $0.36 \mathrm{~m}$; the plasma jet has a length of about $0.7 \mathrm{~m}$ and a diameter increasing from $4 \mathrm{~mm}$ to about $15 \mathrm{~cm}$. The plasma condition is kept constant: arc current $45 \mathrm{~A}$, arc voltage $100 \mathrm{~V}$, background pressure $40 \mathrm{~Pa}$, and argon flow 3.5 standard liters per minute. Details can be found in Refs. [2,7]. Before turning to the results, we will discuss very shortly the principles and diagnostic characteristics of the different methods, as they are well known from literature.

Thomson scattering is the scattering of electromagnetic radiation off free electrons in a plasma. In the classical view, the electron is forced to oscillate with the incident field, after which it becomes a dipole oscillator itself. This dipole radiation can be detected (during Thomson scattering, the wavelength remains unchanged save for Doppler effects, which give $T_{e}$ information) and is proportional to the number of electrons in the detection volume. For Thomson scattering, Nd:YAG (yttrium aluminum garnet) frequency-doubled laser radiation (532 $\mathrm{nm}$ ) is focused on the plasma by a $f=0.5 \mathrm{~m}$ lens, resulting in a beam waist in the focal plane of $100 \mu \mathrm{m}$. The $90^{\circ}$ scattered radiation is transmitted through an optical system, dispersed by a hollow concave grating, and detected by an image-intensified photo diode array. The total Thomson scattered radiation (which can be distinguished from the Rayleigh scattering off bound electrons) is directly proportional to $n_{e}$. Calibration is performed at a known amount of pure argon gas. The apparatus has been used very successfully to determine electron densities and temperatures as well as neutral particle densities in recombining plasma jets $[2,7]$. The dynamic range for $n_{e}$ is $10^{18}-10^{22} \mathrm{~m}^{-3}$, the accuracy around $3-5 \%$. 
The double Langmuir probe is an intrusive method, i.e., it does not leave the plasma undisturbed, as the other techniques described here. By inserting two electrodes (length $7 \mathrm{~mm}$, diameter $0.4 \mathrm{~mm}$ ) in the plasma, the ion saturation current is measured, from which $n_{e}$ can be determined $[8,9]$. Current-voltage characteristics are obtained using a function generator and a personal computer (PC). The PC also determines' $n_{e}$ and $T_{e}$ from the shape of the characteristics. The Langmuir probe is a local method with a large dynamic range $\left(n_{e}\right.$ detection limit around $10^{16} \mathrm{~m}^{-3}$ ).

Optical-emission spectroscopy (OES): The atomic-state distribution function (ASDF) for an excitation system (e.g., argon I) reflects the (non)equilibrium state of a plasma [10]. For the determination of $n_{e}$, the procedure is as follows: using line intensity measurements, an $n_{p} / g_{p}$ vs $I_{p}$ (Boltzmann) plot is constructed, with $n_{p} / g_{p}$ the absolute level population per statistical weight, and $I_{p}$ the ionization potential of the level designated $p$. By assuming the uppermost levels in an atomic system to be in Saha equilibrium with the adjacent ion ground state, $n_{e}$ can be determined as well $[10,11]$. Fitting a straight line through the uppermost levels in a Boltzmann plot gives the electron density (and temperature). A second, more sophisticated method introduces a collisional-radiative (CR) model to describe the level population of all the measured levels. In our recombining case, we used a simple model by Biberman, Vorob'ev, and Yakubov [12], which assumes that the deexcitation from level $p$ downward is equal to the deexcitation ending on level $p$. The model was slightly adjusted to incorporate highly excited states [13]:

$\frac{n_{p}}{g_{p}}=\left[\left(\frac{I_{p}}{k T_{e}}\right]^{3 / 2}+1\right]\left[\frac{n_{e}^{2} h^{3}}{g_{i} g_{e}\left(2 \pi m_{e} k T_{e}\right)^{3 / 2}}\right]$.

In (1), $k$ is Boltzmann's constant; $T_{e}$ is the electron temperature; $h$ is Planck's constant; $g_{i}$ and $g_{e}$ are the statistical weights of the ion ground state and the free electron, respectively; and $m_{e}$ is the electron rest mass. This model is valid for $I_{p} \gg k T_{e}$, whereas for $I_{p} \leq k T_{e}$ Saha equilibrium is assumed; a model fit yields $n_{e}$ and $T_{e}$. A similar model can be applied to ionizing plasmas [12].

In the OES system plasma light is transmitted through an optical system, analyzed by a monochromator (JerrylAsh $0.5 \mathrm{~m}$, resolution $0.16 \mathrm{~nm}$ ), and detected by a cooled $\left(-20^{\circ} \mathrm{C}\right)$ photomultiplier (RCA 31034) $[7,14]$. A number of lines in the argon system are used: 811.5, 763.4 750.4, $703.0,696.5,693.8,591.2,588.9,531.8,518.8,505.0$, and $420.1 \mathrm{~nm}$. Data on these transitions can be found in Refs. $[14,15]$. The optical system is calibrated in an absolute way using a tungsten ribbon lamp (accuracy $<10 \%$ ). Lateral plasma scans are taken using a stepper motordriven rotating mirror. Abel inversion is used to convert the lateral scans into radial $n_{p} / g_{p}$ profiles $[11,16,17]$.

The continuum radiation of this type of plasma is dominated by the electron-ion free-bound radiation. The total continuum emissivity $\epsilon\left(\mathrm{W} \mathrm{m}^{-3} \mathrm{sr}^{-1} \mathrm{~m}^{-1}\right)$ can be written as $[18,19]$

$$
\epsilon=C \frac{\left(n_{e}\right)^{2}}{\lambda^{2} \sqrt{T_{e}}} \xi_{\text {tot }}
$$

with $\lambda$ the wavelength, $C=1.63 \times 10^{-43} \mathrm{~W} \mathrm{~m}^{4} \mathrm{~K}^{1 / 2} \mathrm{sr}^{-1}$, and $\xi_{\text {tot }}=1.7 \pm 0.2$ is the total Biberman factor $[18,19]$. If $T_{e}$ can be estimated (e.g., from a simple Saha fit in a Boltzmann plot, the $T_{e}$ dependence is very weak), $n_{e}$ can be determined.

The continuum part of the spectrum is measured using the OES diagnostic. Continuum radiation was measured in the red $(\lambda=633.5 \mathrm{~nm})$ and blue $(\lambda=468.8 \mathrm{~nm})$ parts of the spectrum. Also in this case, lateral scans are taken and Abel-inverted into radial emissivity data.

The fact that the plasma can be moved inside the vessel without changing significantly facilitates the application of different diagnostic techniques to the same plasma. Great care is taken to make sure that all of the above diagnostics are applied at exactly the same $z$ position (i.e., $\Delta z \approx 0.5 \mathrm{~mm}$ ). With the OES system, measurements are taken at $z=20,40$, and $70 \mathrm{~mm}(z=0$ representing the onset of the expansion, the exit nozzle of the arc). With the Thomson scattering apparatus and the Langmuir probe, data are taken at smaller intervals, covering the entire plasma jet from $z=0$ to $500 \mathrm{~mm}$. A comparison between all four methods will be made at $z=20,40$, and $70 \mathrm{~mm}$.

\section{RESULTS AND DISCUSSION}

Table I gives a compilation of the results. The Thomson scattering data are taken as a standard, as this diag-

TABLE I. A comparison of five methods to determine $n_{e}$ in recombining plasmas: TS: Thomas scattering; CRM: collisional radiative model; Saha: Saha fit through uppermost levels; Cont.: Continuum measurements averaged for blue and red part of the spectrum; Probe: double Langmuir probe. In the third main column, $n_{e}^{*}$ represents $n_{e}$ values calculated using $T_{e}$ obtained from Thomson scattering measurements.

\begin{tabular}{|c|c|c|c|c|c|c|c|c|c|}
\hline \multirow{2}{*}{ Method $z(\mathrm{~mm})$} & \multicolumn{3}{|c|}{$n_{e}\left(10^{19} \mathrm{~m}^{-3}\right)$} & \multicolumn{3}{|c|}{$\Delta n_{e}(\%)$} & \multicolumn{3}{|c|}{$n_{e}^{*}\left(10^{19} \mathrm{~m}^{-3}\right)$} \\
\hline & 20 & 40 & 70 & 20 & 40 & 70 & 20 & 40 & 70 \\
\hline TS & 6.5 & 1.8 & 4.6 & 15 & 7.5 & 8 & & & \\
\hline CRM & 5.0 & 1.7 & 8.4 & 50 & 50 & 50 & 5.0 & 2.0 & 4.5 \\
\hline Saha & 3.7 & 4.2 & 9.8 & 60 & 60 & 60 & 3.5 & 2.8 & 4.5 \\
\hline Cont. & 5.3 & 2.2 & 3.8 & 30 & 30 & 30 & & & \\
\hline Probe & 6.0 & 5.4 & 4.5 & 15 & 15 & 15 & & & \\
\hline
\end{tabular}




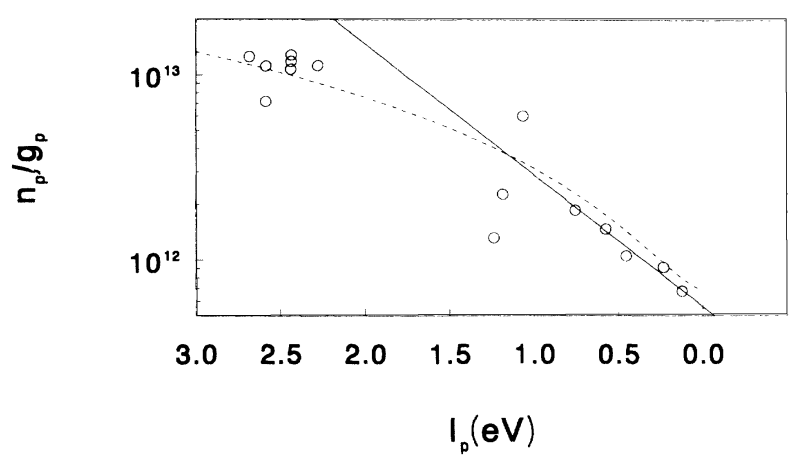

FIG. 2. An example of a Boltzmann plot: $n_{p} / g_{p}$ vs $I_{p}$, constructed using the OES measurements. The data are taken at $z=70 \mathrm{~mm}$. The dotted line represents a fit with a collisional radiative model, whereas the drawn line is a simple straight line fit through the uppermost levels, assuming these to be in Saha equilibrium with the adjacent continuum.

nostic requires no assumptions on plasma equilibrium, etc. The margins of error are established by adding the relative errors in the measurement and data-acquisition procedure $(5 \%$ [2]) to those introduced by plasma reproducibility. Thomson scattering is the only diagnostic which is intrinsically accurate enough to render these day-to-day plasma changes observable.

Figure 2 gives an example of a Boltzmann plot at $z=70$ $\mathrm{mm}$, with both a collisional-radiative-model (CRM) fit and a simple Saha fit. The margins of error indicated in Table I are a result of (a) plasma reproducibility errors (10\%); (b) errors in the calibration $(<10 \%)$; (c) errors in the transition probabilities $(25-50 \%,[15])$, the Poisson statistics (usually <10\%); and (d) errors in the Abelinversion procedure (a few \%).

The margins of error for the results calculated from the continuum emissivity are due to (a) plasma reproducibility errors $(10 \%)$, (b) calibration errors $(<10 \%)$, (c) errors in the Abel-version procedure (a few percent), and (d) the Poisson statistics (around $30 \%$, as the signal is very weak).

The double-probe measurements need some additional explanation. Even though the statistical errors (indicated in Table I) are rather small, a large difference with the Thomson data is obtained at $z=40 \mathrm{~mm}$, where the stationary shock front is situated [2]. This may be caused by the disturbance of the flow pattern by the probe insertion, which is most drastic within the shock, where gradients are large. Furthermore, the probe area $\left(2 \times 7 \mathrm{~mm}^{2}\right)$ may be too large to fully resolve the shock feature, introducing systematic errors. The values outside the shock region are in rather good agreement with the Thomson scattering data. This is clear in Fig. 3, which shows a comparison of Thomson scattering and probe data for a range of axial positions. The systematic deviation downward for $z>150 \mathrm{~mm}$ is a consequence of a poorly determined $T_{e}$, resulting in a low- $n_{e}$ value. When the Thom-

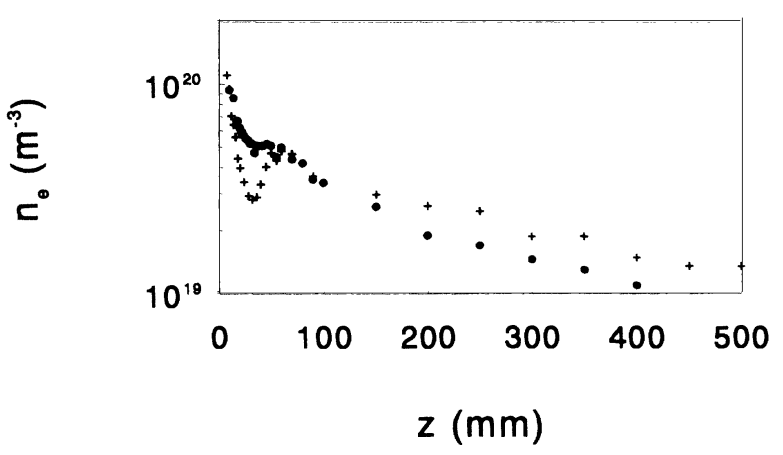

FIG. 3. A comparison between Thomson scattering (crosses) and Langmuir probe (circles) data: the electron density on the axis of the plasma jet vs the distance from the onset of the expansion. The general agreement is good whereas in the shock region (around $z=40 \mathrm{~mm}$ ) a deviation is observed: insertion of the probe evidently disturbs the flow pattern significantly at these axial positions.

son scattering $T_{e}$ [2] is used to correct for these errors, an agreement within $10 \%$ is reached for $z>150 \mathrm{~mm}$. The discrepancy at the shock position, however, is not solved in this manner [20].

The Thomson scattering values for $T_{e}$ can also be used to correct the $n_{e}$ values as determined from the OES measurements, thus eliminating one of the major drawbacks of the OES method. The $T_{e}$ values are $0.17,0.24$, and $0.26 \mathrm{eV}$ at $z=20,40$, and $70 \mathrm{~mm}$, respectively [14] The results are given in the last column of Table I. The $n_{e}$ values, thus obtained with the Saha method, show a much better agreement with Thomson scattering data. For the CR model, the value at $z=70 \mathrm{~mm}$ is brought in line with Thomson data. This shows that, particularly when determining $n_{e}$ using Saha, the error in $T_{e}$ is a dominant factor. The continuum measurements show no significant difference when Thomson scattering $T_{e}$ values are used.

In conclusion we can state that there are some good alternatives to Thomson scattering for the determination of $n_{e}$, if accuracies around $30 \%$ are permissible. Probe measurements are an alternative, especially since the detection limit is low: around $10^{16} \mathrm{~m}^{-3}$. A drawback may be the fact that flow patterns are disturbed when the probe is inserted. Both the continuum results and the CRM fits yield results in good agreement with Thomson scattering data. A simple Saha fit proved to be adequate only to give an indication for the order of magnitude of $n_{e}$ (i.e., within a factor of $2-3$ ).

\section{ACKNOWLEDGMENTS}

The authors would like to thank A. Griguoli, H. M. M. de Jong, and M. J. F. van der Sande for their skillful assistance during the measurements. 
[1] J. Sheffield, Plasma Scattering of Electromagnetic Radiation (Academic, New York, 1975).

[2] M. C. M. van de Sanden, J. M. de Regt, G. M. Janssen, J. A. M. van der Mullen, D. C. Schram, and B. van der Sijde, Rev. Sci. Instrum. 63, 3369 (1992).

[3] J. J. Beulens, G. M. W. Kroesen, D. C. Schram, P. K. Bachman, H. Lydtin, and D. U. Wiechert, Surf. Mod. Tech. III, 69 (1989).

[4] A. T. M. Wilbers, G. J. Meeusen, M. Haverlag, G. M. W. Kroesen, and D. C. Schram, Thin Solid Films 204, 59 (1991).

[5] R. F. G. Meulenbroeks, D. C. Schram, L. J. M. Jaegers, and M. C. M. van de Sanden, Phys. Rev. Lett. 69, 1379 (1992).

[6] M. C. M. van de Sanden, J. H. de Regt, and D. C. Schram, Phys. Rev. E 47, 2792 (1993).

[7] M. C. M. van de Sanden, Ph.D. thesis, Eindhoven University of Technology, 1991.

[8] L. Schott, in Plasma Diagnostics, edited by W. LochteHoltgreven (North-Holland, Amsterdam, 1968).

[9] M. J. de Graaf, R. Severens, R. P. Dahiya, M. C. M. van de Sanden, and D. C. Schram, Phys. Rev. E 48, 2098 (1993).

[10] J. A. M. van der Mullen, Phys. Rep. 191, 109 (1990).

[11] G. J. Meeusen, E. A. Ershov-Pavlov, R. F. G. Meulenbroeks, M. C. M. van de Sanden, and D. C. Schram, J.
Appl. Phys. 71, 4156 (1992).

[12] L. M. Biberman, V. S. Vorob'ev, and I. T. Yakubov, Kinetics of Nonequilibrium Low Temperature Plasmas (Plenum, New York, 1987).

[13] D. A. Benoy, J. A. M. van der Mullen, M. C. M. van de Sanden, B. van der Sijde, and D. C. Schram, J. Quantum Spectrosc. Radiat. Transfer 49, 129 (1993).

[14] R. F. G. Meulenbroeks, A. J. van Beek, A. J. G. van Helvoort, M. C. M. van de Sanden, and D. C. Schram (unpublished).

[15] W. L. Wiese, M. W. Smith, and B. M. Miles, Atomic Transition Probabilities, Report No. NSRDS-NBS22, (Nat. Bur. Std. Washington, D.C., 1969).

[16] S. R. Deans, The Radon Transform and Some of its Applications (Wiley-Interscience, New York, 1983).

[17] C. A. Kak and M. Slaney, Principles of Computerized Tomographic Imaging (IEEE, New York, 1988).

[18] V. M. Lelevkin, D. K. Otorbaev, and D. C. Schram, Physics of Nonequilibrium Plasmas (North-Holland, Amsterdam, 1992), Chap. 8.

[19] A. T. M. Wilbers, G. M. W. Kroesen, C. J. Timmermans, and D. C. Schram, J. Quantum Spectrosc. Radiat. Transfer 45, 1 (1991).

[20] Z. Qing, M. J. de Graaf, D. K. Otorbaev, M. C. M. van de Sanden, and D. C. Schram (unpublished). 


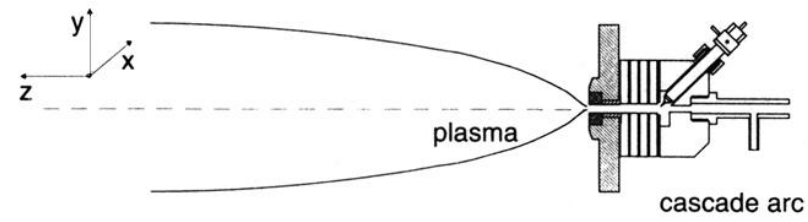

FIG. 1. The cascaded arc experiment. A thermal plasma at subatmospheric pressure is created in a continuously operated dc arc, consisting of three cathodes, three electrically isolated copper plates, and an anode plate. All the parts are water cooled. The plasma expands through the nozzle into a lowpressure $(40 \mathrm{~Pa})$ vessel, creating a supersonically expanding plasma jet. 